\title{
Structure, Design, and Mechanics of a Pop-Up Origami with Cuts
}

\author{
Taiju Yoneda, ${ }^{1}$ Yoshinobu Miyamoto, ${ }^{2}$ and Hirofumi Wada ${ }^{1, *}$ \\ ${ }^{1}$ Department of Physical Sciences, Ritsumeikan University, Kusatsu, Shiga 525-8577, Japan \\ ${ }^{2}$ Department of Architecture, Aichi Institute of Technology, Toyota, Aichi 470-0392, Japan
}

(Received 20 August 2021; revised 10 December 2021; accepted 5 January 2022; published 23 February 2022)

\begin{abstract}
The rotational erection system (RES) is an origami-based design method to generate a three-dimensional (3D) structure from a planar sheet. Its rotational and translational kinematics are fully encoded in the form of prescribed cuts and folds. Here we characterize the mechanical properties of a threefold symmetric RES by combining finite element analysis and a physical experiment. Whereas the crease and cut patterns imply that the system is not rigid foldable, we demonstrate that plate bending creates a physical route connecting the two energetically separated configurations. The elasticity of the RES allows the RES to morph into a 3D shape via a snapthrough transition. We show that the energy barrier for bistability is independent of the entire span of the structure, and depends solely on its aspect ratio. We also indicate that the standing RES has structural rigidity and resilience, owing to its unique self-locking mechanism, indicating its superior load-bearing performance in a range of applications. The present study clarifies the fundamental actuation mechanism of an origami-based deployable structure extended with chiral patterned cuts, which facilitates a way to use the optimally designed RES in a variety of human-made systems.
\end{abstract}

DOI: 10.1103/PhysRevApplied.17.L021004

\section{INTRODUCTION}

An origami-inspired design facilitates the creation of a three-dimensional (3D) structure from a planar sheet or membrane [1-9]. As a folded 3D configuration involves self-contact and overlap and the surface area must be conserved owing to the inextensibility of the sheet, its lateral extent usually diminishes such that the structure grows vertically. This inherent feature of origami underlies the prominent foldability of large membranes that are utilized in a range of natural [10-15] and human-made systems [16-18].

In contrast, kirigami is a design method used to make a paper sheet highly stretchable by periodically introducing free boundaries, that is, cuts and holes $[19,20]$. In a typical kirigami design, a sheet is free of any overlap or selfcontact; however, it is mechanically monostable, meaning that its extended state is stable only in the presence of external loading. Thus, it is interesting to create a multistable geometric design in which a single flat sheet morphs vertically into a stable 3D structure, while retaining its lateral extent. Various kirigami-based pop-up designs for 3D

\footnotetext{
*hwada@fc.ritsumei.ac.jp
}

Published by the American Physical Society under the terms of the Creative Commons Attribution 4.0 International license. Further distribution of this work must maintain attribution to the author(s) and the published article's title, journal citation, and DOI. patterns that can be actuated by global (tensile) loading have recently been proposed and studied [21-24]. Strategies for creating $3 \mathrm{D}$ microstructures for photonics and flexible electronics are also based on similar concepts. More specifically, such 3D microstructures are designed to be actuated by global buckling compression [25]. Indeed, an elaborate combination of folds and cuts, called "orikirigami," is a promising method to develop a class of shape-shifting materials [26-34].

The RES developed in Refs. [35,36] provides a unique example of a $2 \mathrm{D}$ to $3 \mathrm{D}$ transformation from two dimensions to three without any global edge loading. RES is bistable and reversibly transforms into a stable 3D structure from a planar sheet with prescribed cuts and fold lines, by means of only stretches and/or twists applied at selected points (Fig. 1 and Movie S1 in the Supplemental Material [37]). Besides supporting its own weight, the resulting shape can also support an external load, implying that the RES would be an efficient production method for industrial, artistic, and architectural uses. It is crucial to obtain a quantitative and predictive understanding of the mechanical and kinematic properties of an RES to control and optimize RES functions.

In this study, we first present numerical simulations based on finite element analysis (FEA) to characterize the physical properties of the RES. Compared to existing origami simulators [38], our FEA simulation is physically more informative, primarily because the FEA simulation accurately accounts for out-of-plane elastic bending and 


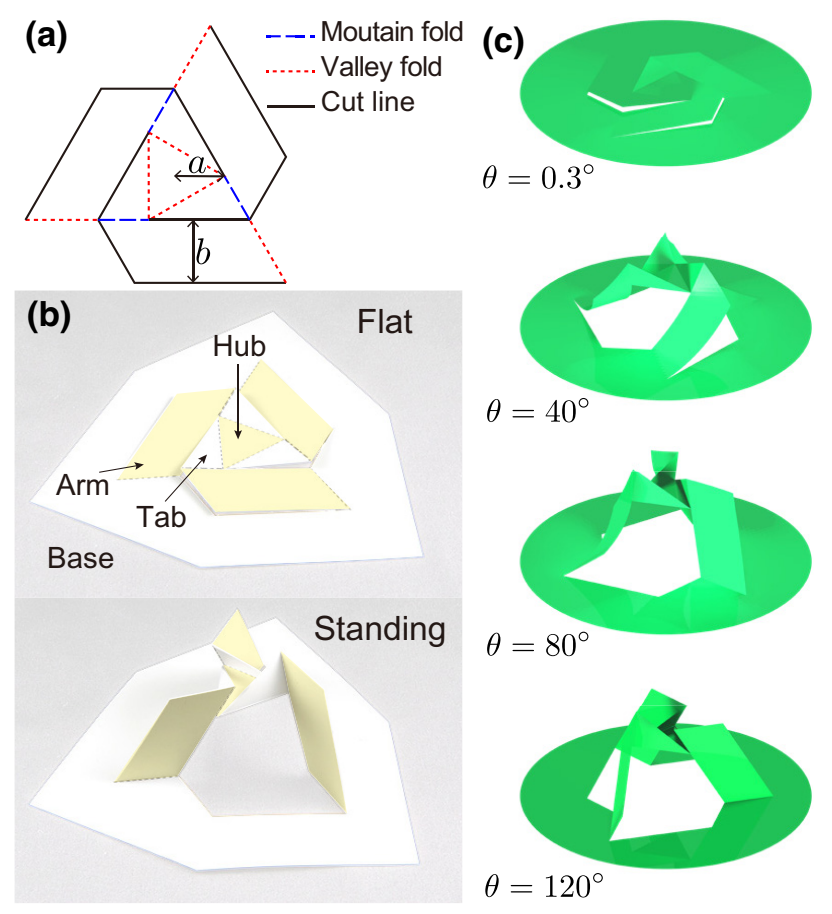

FIG. 1. An overview of the RES. (a) Mountain and valley creases, and cut lines, with the definition of geometric lengths $a$ and $b$. (b) Photographs of a paper RES with the definitions of the names of the specific parts, in (almost) flat and standing configurations. (c) Sequence of snapshots of the RES generated from our finite element simulations (see the text for details).

twisting, and in-plane stretching of all the plates constituting the entire RES. Our FEA prediction is then quantitatively verified by a physical experiment using a real RES engineered from synthetic paper.

\section{SIMULATION AND EXPERIMENTS}

While a variety of multistable RES designs exist, the main focus of this work is a threefold symmetric RES, as illustrated in Fig. 1(a) that embodies an essential mechanism common to a family of more complex RES designs [39]. Because the RES morphs into a 3D shape as it is rotated in the vertical direction (defined as the $z$-axis), we characterize its configuration using its height $z$ and rotational angle $\theta$ [35]. It should be noted that rotational actuation is an integral element in the RES design, which allows it to take up additional lengths from a flat, inextensive sheet to grow vertically. In this study, the physical RES comprises sufficiently stiff plates (explained below); thus, the effects of gravity are negligible.

Structurally, an RES consists of four parts: the base, arms, tabs, and hub (see Fig. 1). Simply counting the degrees of freedom of our RES suggests that it is overconstrained and not rigid foldable [37,40-43]. In contrast, it is possible to readily actuate the RES composed of real materials by twisting the hub with respect to the base.
We conducted an FEA simulation using the commercial software ABAQUS (Dassault Systems) to quantify this unique morphing pathway. The technical details are summarized in the Supplemental Material [37]. We began our computation with a slightly lifted shape from a flat plane and simulated a complete cycle. As shown later, an ideal RES accompanies buckling of its arms out of its flat state. Thus, we introduced a perturbation as a form of initial lift to induce coordinated buckling between the three arms.

Furthermore, we conducted a physical experiment to verify the simulation results. The RES with dimensions $a=25 / \sqrt{3} \mathrm{~mm}$ and $b=18 \mathrm{~mm}$ was processed at Fuji Toso Kogyo Co., Ltd. using YUPO synthetic paper $t=$ $0.5 \mathrm{~mm}$ thick. YUPO paper has a well-defined anisotropy in its bending stiffness owing to the manufacturing process. Therefore, we conducted an independent mechanical test to determine the YUPO paper's direction-averaged bending stiffness per unit width of $0.022 \mathrm{~N} \mathrm{~m}$ (see the Supplemental Material [37]). This amounts to an effective Young's modulus of $E=1.9 \mathrm{GPa}$, given the Poisson ratio $v=0.3$, which may be valid for the major component of YUPO paper, polypropylene. We introduced a half-cut on a crease line that can be regarded as a freely rotatable hinge [44]. For the torque and force measurements, we developed a home-built experimental system that is detailed in the Supplemental Material [37]. In the experiment, we only investigated the folding (i.e., standing to flat) process, in which the twisting angle $\theta$ was quasistatically decreased by $5^{\circ}$, from $120^{\circ}$. The mechanical response of the deployment process (flat to standing) was explored using FEA.

\section{RESULTS}

In Fig. 2(b), we plot the rescaled experimental torque $M b / B$, where $B=E t^{3} b /\left[12\left(1-v^{2}\right)\right]$ is the bending modulus per arm of width $b$ [45], combined with the FEA simulation data. Overall, the numerical and experimental data exhibit similar trends, confirming the validity of the two independent approaches.

First, we focus on the $\theta \ll 1$ region, where the RES is activated from the flat configuration. For an ideal RES studied in the simulation, the change in shape begins with the buckling of the three arms. Accordingly, the torque increases discontinuously at $\theta=0$, from zero to $M_{c+} \approx$ $2.5 B / b$ [Fig. 2(b)]. Suppose a thin strip of length $L$, thickness $t$, and width $b$, subjected to an external force $f$ is applied at one end [45]. The strip buckles at $f_{c} \approx \alpha B / L^{2}$, where $\alpha$ is a numerical prefactor of the order of $1-10$ sensitively depending on the boundary conditions and geometry of the strip [46]. Assuming threefold symmetry, the buckling torque is $M_{c+} \sim 3 f_{c} a \sim 3 \alpha B a / L^{2}$. For the arm of the RES considered here, $L \approx 2 a$, we predict $M_{c+} b / B \approx$ $(3 \alpha / 4) b / a$. In Fig. 2(e), we plot $M_{c+}$ for different values of $b / a$ obtained from the simulations, confirming 
(a)
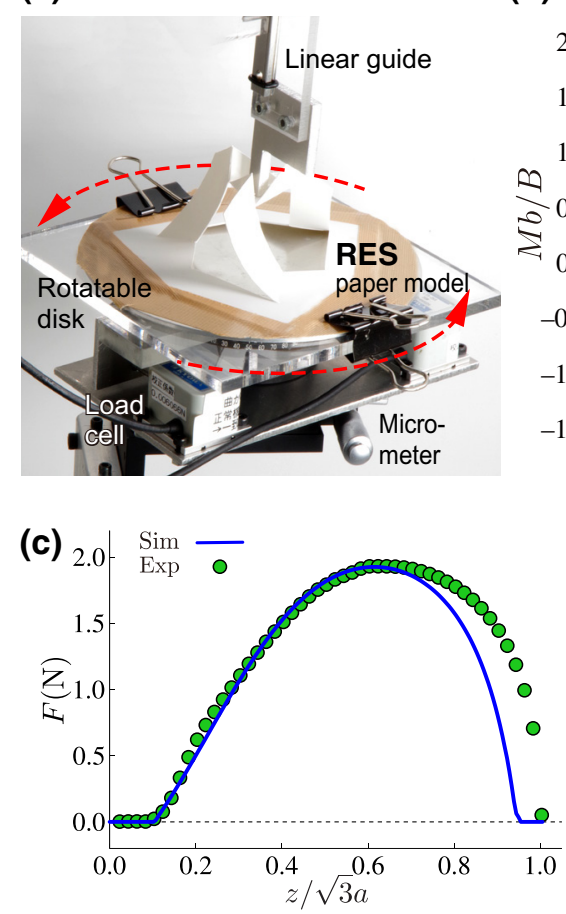
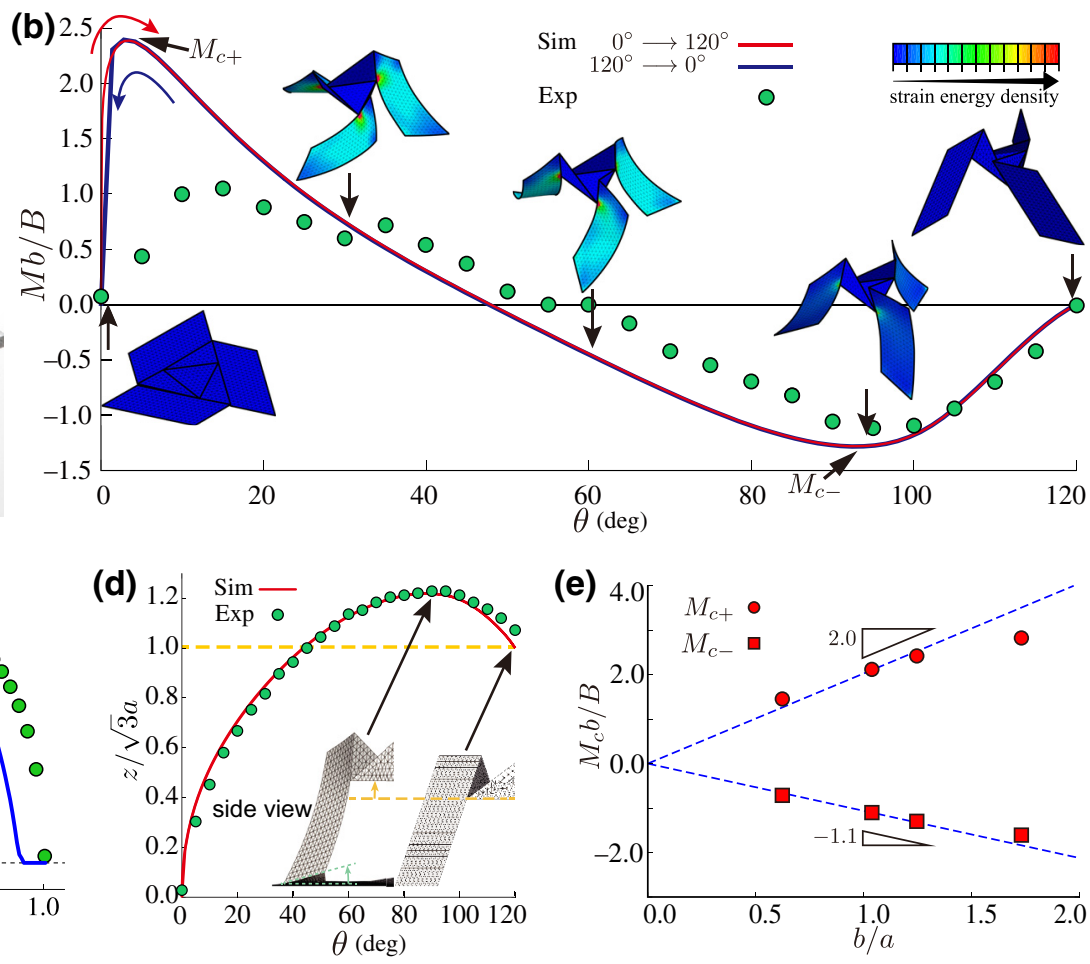

FIG. 2. Experimental and numerical results for our RES mechanics and geometry. (a) Experimental system to measure RES torque response. (b) Rescaled torque $M b / B$ plotted as a function of the rotational angle $\theta$ (in degrees) obtained from the experiment (filled green symbols) and the FEA simulations (solid red and blue lines). The corresponding FEA snapshots were displayed simultaneously. The color map shows the total strain energy density. (c) Force versus displacement curves from the experiment and FEA simulations. (d) Rescaled height (defined as the vertical distance between the base and the hub), $z /(\sqrt{3} a)$, plotted as a function of $\theta$, in a similar manner to (b). Inset: corresponding snapshots obtained from FEA simulations. The horizontal yellow line represents hub equilibrium height. (e) Peak values of the torque in (b) (indicated by $M_{c+}$ and $M_{c-}$ ) are functions of $a / b$. Dashed lines are fits to the data based on the prediction of the scaling theory (refer to the text).

that $M_{c+} b / B \sim b / a$. A deviation was only observed for $b / a>1$, for which a narrow strip assumption is no longer valid. Fitting the data in Fig. 2(e) allows us to determine $\alpha \simeq 2.7$, which is a reasonable value for the critical buckling force [46]. In contrast to the simulated RES, the arms of our physical RES are not entirely flat. In practice, the arms have slight permanent curvature. Thus, the physical RES stands up from the (nearly) flat configuration without any buckling, leading to a smooth experimental torque curve with a reduced slope and peak value, as illustrated in Fig. 2(b). We also simulated an RES whose hub was lifted vertically by an amount comparable to the experimental one in its initial configuration to corroborate the aforementioned aspects (Fig. S7 in the Supplemental Material [37]). The FEA indicates that both the slope and peak decrease as the initial lift increases, confirming the validity of our argument.

At the maximum torque the slope becomes negative with increasing $\theta$, and at $\theta=\theta_{c} \approx 50^{\circ}$ the torque crosses the unstable equilibrium point where $M=0$, at which point it is likely to snap. We conducted a uniaxial stretching experiment to confirm the outcomes by allowing the
RES to rotate freely during extension. (For details, see the Supplemental Material [37], in particular Movie S1.) The experimental force $(F)$ versus displacement $(z)$ curve was compared with the FEA simulation, showing good agreement up to the unstable region $(d F / d z<0)$ [Fig. 2(c)]. In the FEA, $F$ becomes zero at $z_{c} /(\sqrt{3} a) \approx 0.95$, which corresponds to $\theta_{c} \approx 50^{\circ}$, as shown in Fig. 2(d). Owing to the viscoplasticity of the hinges in our YUPO-based paper model, the experimental snap point shifts toward larger $z$. (For more details, see the Supplemental Material [37].) Considering the complexity of a real RES model, our numerical and experimental investigations of the torque and force responses are consistent overall, confirming the presence of the snapping transition at $\theta_{c}=$ $50^{\circ}-60^{\circ}$.

We now focus on the behavior around the position at $\theta=120^{\circ}$. We define $\varphi$ as $\theta=120^{\circ}-\varphi$ to highlight the extent of departure from it. We observe in Fig. 2(b) that the magnitude of the torque increases almost linearly with $\varphi$ for $0<\varphi<20^{\circ}$. Remarkably, the height of the RES also increased for this range of $\varphi$ [Fig. 2(d)]. As the hub begins to rotate, its arms are bent and simultaneously pushed 
outwards. As the arms are tilted with respect to the flat base, the hub must be initially lifted upward. Refer to Fig. 2(d) and Fig. S6 in the Supplemental Material [37]. Note that the base slightly leaves the ground during the process. The compliance of the base significantly lowers the risk of RES damage, particularly in the vicinity of the joints to the base; however, compliance is not essential for the kinematics illustrated in Fig. 2(d). The unique kinematics that "locks" the 3D configuration of the RES underlies the structural rigidity of the RES investigated below.

\section{DISCUSSION}

In Fig. 2(b), the torque response was fully reversible. However, the energy landscape around $\theta=0^{\circ}$ is more complicated than that expected from Fig. 2(b). The RES is allowed to take on multiple kinematic pathways at $\theta=0^{\circ}$, most of which lead to a partial transformation. For the RES to be on the "right" path at $\theta=0^{\circ}$, all three arms need to buckle in the same direction synchronously, allowing the hub to start moving vertically, keeping its horizontality. The up-down symmetry is broken externally if a vertical tensile force is applied to the hub, which readily induces synchronized buckling, exactly in the same manner in which we involuntarily play with the paper model manually. Similarly, if the arms have a small initial curvature in a similar direction, it will assist the entire structure deform smoothly without instabilities, as observed in Fig. 2(b). Collectively, a weak distortion intrinsic in structures made of real physical materials, combined with visually guided manipulations manually, can drastically simplify the morphing energy landscape, which then enables the RES to perform as intended, with a stable and reproducible cyclic actuation. This feature will be a fairly generic consequence that is most likely valid for a wide class of functionally foldable thin structures, beyond the specific example studied here.

Evidently, the RES jumps from one stable state to another by bending its three arms elastically. A distributed curvature over the surface of the arm functions as a "virtual crease," much as for origami with a square-twist pattern [6]. The bending energy connects a kinematic pathway between the flat and standing configurations that are otherwise isolated in the configuration space [47]. We quantified the energy barrier for bistability based on the torque response, as illustrated in Fig. 2(b), according to $W=\int M(\theta) d \theta$, which is plotted in Fig. 3 as a function of $\theta$. We reproduced an expected double-well potential with two stable states of similar energy values. According to the scaling argument given previously, it can be concluded that the energy barrier for the shape transition, $U$, is given by

$$
U \sim \frac{E t^{3} b}{a}
$$

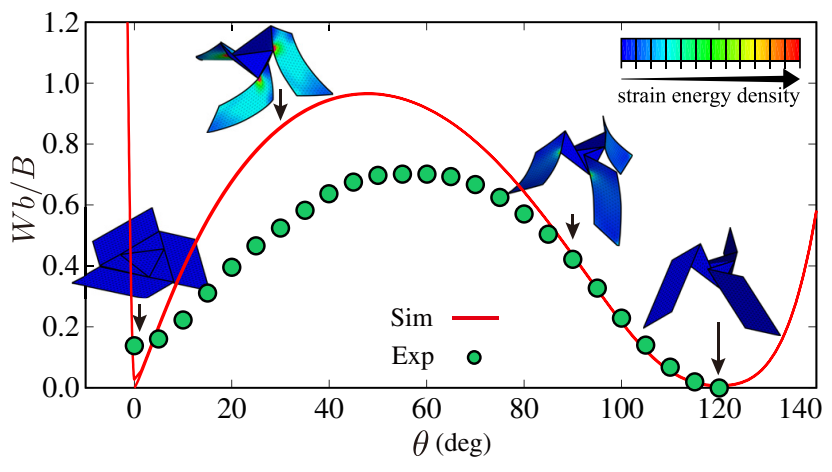

FIG. 3. The energy profiles for the bistability of the RES, reconstructed from the torque versus angle curves as illustrated in Fig. 2(b), obtained from the simulations and experiments. In the simulation data, the parameter range of $\theta$ was extended to include $\theta<0^{\circ}$ and $\theta>120^{\circ}$.

which is independent of the entire span of the structure; the energy barrier solely depends on the ratio $b / a$, indicating that the bistability of RES is essentially scale-independent. The results confirm the validity of the bar-hinge (truss) model, in which a smooth deflection of the arm was substituted with a localized bending stiffness across the virtual crease independent of the length of arm $a$.

Having characterized the morphological aspects of the RES, we performed a compression test to investigate the load-displacement curves (Fig. 4). We found that a vertical compression force induces the twisting of the RES beyond $\theta=120^{\circ}$, thereby rigidifying the entire structure. Therefore, the RES resists the flattening transition, recovering its original configuration immediately after the loading is removed, as in an ordinary elastic spring [48]. Moreover, the restoring force increases with an increase in the vertical displacement, where the arms buckle and deflect accordingly. In fact, the flattening transition (i.e., collapse) of the RES never occurs with compressive loading only. This
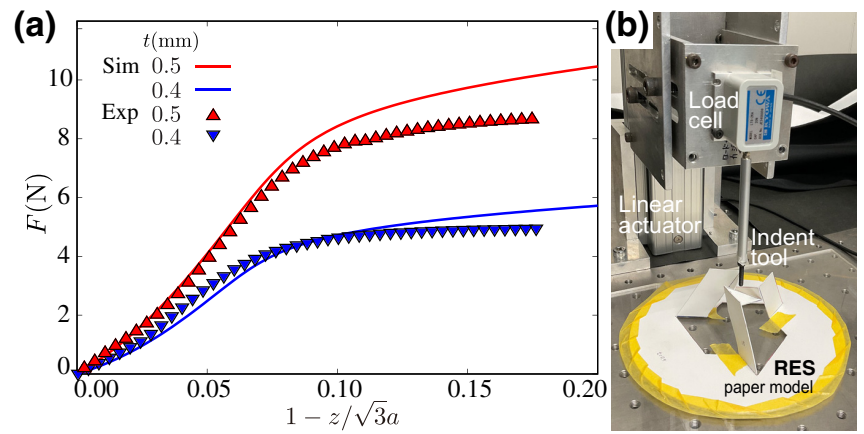

FIG. 4. Compression test of the RES in the standing configuration. (a) Load versus displacement curves obtained from the experiment (symbols), and the FEA simulations (solid lines) for different plate thicknesses $t=0.4 \mathrm{~mm}$ (blue) and $0.5 \mathrm{~mm}$ (red). (b) Experimental setup for force measurement. 


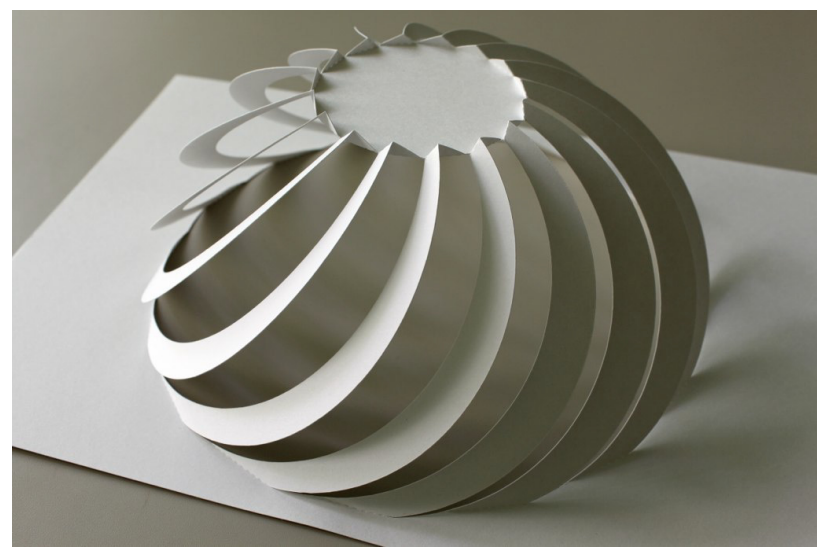

FIG. 5. A paper model of an RES-based hemispherical dome. A collection of pleasing photographs of a variety of RES-based designs can be found in the Supplemental Material [37].

was deduced based on the kinematics of the RES, illustrated in Fig. 2(d), where the RES first has to increase its height to reach the flat configuration. The RES has to be pulled upward first (allowed to rotate freely), and then pushed downward, which is clearly an impossible mode with a pure compressive load only. The built-in self-locking mechanism is illustrated in Fig. 2(d), which indicates the structural rigidity of the standing RES shown in Fig. 4(a).

In conclusion, the actuation of a rotationally patterned 3D structure from a planar sheet represents a robust design principle in a variety of engineering systems [25]. A variety of elaborate planar patterns of cuts and folds form a family of RESs with different 3D architectures and topologies, including domes (Fig. 5) and multistage towerlike configurations [35]. For more details, refer to the Supplemental Material [37]. These complex designs share many geometrical and mechanical principles that have been revealed for the simplest threefold symmetric model. A comprehensive study of the design space and optimization of RES will be a critical subject in the future. The scale-free and tunable nature of RES bistability will potentially be useful in a wide range of applications, including switching devices, energy-adsorbing mechanical systems, and one-step construction in architectures. Our proof-ofconcept study clarifies the fundamental actuation mechanism of an origami-based deployable structure extended with cuts and determines the use of an optimally designed RES in a range of artificial systems.

\section{ACKNOWLEDGMENTS}

Financial support from JSPS KAKENHI (Grants No. $18 \mathrm{~K} 13519$ (to H.W.) and No. 20K12518 (to Y. M.)) and Grant-in-Aid for JSPS Research Fellow (DC1, No. 19J22381 (to T.Y.)) is gratefully acknowledged.
[1] E. D. Demaine, M. L. Demaine, D. Koschitz, and T. Tachi, in Proc. IABSE-IASS Symp. Taller, Longer, Light. (IABSEIASS 2011, 2011).

[2] T. Tachi, Designing freeform origami tessellations by generalizing resch's patterns, J. Mech. Des. 135, 111006 (2013).

[3] A. Lebée, From folds to structures, a review, Int. J. Space Struct. 30, 55 (2015).

[4] M. Schenk and S. D. Guest, Geometry of miura-folded metamaterials, Proc. Natl. Acad. Sci. USA 110, 3276 (2013).

[5] J. L. Silverberg, A. A. Evans, L. McLeod, R. C. Hayward, T. Hull, C. D. Santangelo, and I. Cohen, Using origami design principles to fold reprogrammable mechanical metamaterials, Science 345, 647 (2014).

[6] J. L. Silverberg, J.-H. Ha, A. A. Evans, B. Liu, T. Hull, C. D. Santangelo, R. J. Lang, R. C. Hayward, and I. Cohen, Origami structures with a critical transition to bistability arising from hidden degrees of freedom, Nat. Mater. 14, 389 (2015).

[7] F. Lechenault and M. Adda-Bedia, Generic Bistability in Creased Conical Surfaces, Phys. Rev. Lett. 115, 235501 (2015).

[8] H. Yasuda and J. Yang, Reentrant Origami-Based Metamaterials with Negative Poisson's Ratio and Bistability, Phys. Rev. Lett. 114, 185502 (2015).

[9] J. A. Faber, A. F. Arrieta, and A. R. Studart, Bioinspired spring origami, Science 359, 1386 (2018).

[10] H. Kobayashi, B. Kresling, and J. F. V. Vincent, The geometry of unfolding of tree leaves, Proc. R. Soc. B 265, 147 (1998).

[11] L. Mahadevan and S. Rica, Self-organized origami, Science 307, 1740 (2005).

[12] C. Py, P. Reverdy, L. Doppler, J. Bico, B. Roman, and C. N. Baroud, Capillary Origami: Spontaneous Wrapping of a Droplet with an Elastic Sheet, Phys. Rev. Lett. 98, 156103 (2007).

[13] M. J. Harrington, K. Razghandi, F. Ditsch, L. Guiducci, M. Rueggeberg, J. W. C. Dunlop, P. Fratzl, C. Neinhuis, and I. Burgert, Origami-like unfolding of hydro-actuated ice plant seed capsules, Nat. Commun. 2, 2 (2011).

[14] W. T. M. Forbes, The wing folding pattern of the coleoptera, J. NY Entomol. Soc. 24, 91 (1926).

[15] K. Saito, S. Nomura, S. Yamamoto, R. Niyama, and Y. Okabe, Investigation of hindwing folding in ladybird beetles by artificial elytron transplantation and microcomputed tomography, Proc. Natl. Acad. Sci. USA 114, 5624 (2017).

[16] K. Miura, Method of packaging and deployment of large membranes in space, Tech. Rep. (Inst. Space Astronaut Sci., 1985).

[17] J. T. Bruton, T. G. Nelson, T. K. Zimmerman, J. D. Fernelius, S. P. Magleby, and L. L. Howell, Packing and deploying soft origami to and from cylindrical volumes with application to automotive airbags, R. Soc. Open Sci 3, 160429 (2016).

[18] K. Kuribayashi, K. Tsuchiya, Z. You, D. Tomus, M. Umemoto, and M. Sasaki, Self-deployable origami stent grafts as a biomedical application of ni-rich tini shape memory alloy foil, Mater. Sci. Eng. A 419, 131 (2005).

[19] Z. Qi, D. K. Campbell, and H. S. Park, Atomistic simulations of tension-induced large deformation and 
stretchability in graphene kirigami, Phys. Rev. B 90, 245437 (2014).

[20] M. Isobe and K. Okumura, Initial rigid response and softening transition of highly stretchable kirigami sheet materials, Sci. Rep. 6, 24758 (2016).

[21] A. Rafsanjani and K. Bertoldi, Buckling-Induced Kirigami, Phys. Rev. Lett. 118, 084301 (2017).

[22] M. A. Dias, M. P. McCarron, D. J. Rayneau-Kirkhope, P. Z. Hanataka, D. K. Campbell, H. S. Park, and D. P. Holmes, Kirigami actuators, Soft Matter 13, 9087 (2017).

[23] Z. Liu, H. Du, J. Li, L. Lu, Z.-Y. Li, and N. X. Fang, Nanokirigami with giant optical chirality, Sci. Adv. 4, eaat4436 (2018).

[24] M. H. Kaspersen, S. Hines, M. Moore, M. K. Rasmussen, and M. A. Dias, in Procceedings of the 2019 on Designing Interactive Systems Conference (2019), p. 935.

[25] Y. Zhang, F. Zhang, Z. Yan, Q. Ma, X. Li, Y. Huang, and J. A. Rogers, Printing, folding and assembly methods for forming $3 \mathrm{~d}$ mesostructures in advanced materials, Nat. Rev. Mater. 2, 1 (2017).

[26] T. Nojima and K. Saito, Development of newly designed ultra-light core structures, JSME Int. J. A 49, 38 (2006).

[27] K. Saito, F. Agnese, and F. Scarpa, A cellular kirigami morphing wingbox concept, J. Int. Mat. Sys. Struct. 22, 935 (2011).

[28] T. Castle, Y. Cho, X. Gong, E. Jung, D. M. Sussman, S. Yang, and R. D. Kamien, Making the cut: Lattice Kirigami Rules, Phys. Rev. Lett. 113, 245502 (2014).

[29] R. M. Neville, F. Scarpa, and A. Pirrera, Shape morphing kirigami mechanical metamaterials, Sci. Rep. 6, 31067 (2016).

[30] K. Bertoldi, V. Vitelli, J. Christensen, and M. van Hecke, Flexible mechanical metamaterials, Nat. Rev. Mater. 2, 17066 (2017).

[31] A. Rafsanjani, Y. Zhang, B. Liu, S. M. Rubinstein, and K. Bertoldi, Kirigami skins make a simple soft actuator crawl, Sci. Robotics 3, eaar7555 (2018).

[32] D. P. Holmes, Elasticity and stability of shape-shifting structures, Curr. Opin. Colloid Interface Sci. 40, 118 (2019).

[33] M. Liu, L. Domino, and D. Vella, Tapered elastica as a route for axisymmetric morphing structures, Soft Matter 16, 7739 (2020).

[34] Z. Zhai, L. Wu, and H. Jiang, Mechanical metamaterials based on origami and kirigami, Appl. Phys. Rev. 8, 041319 (2021).

[35] Y. Miyamoto, in Origmai 6: The 6th International Meeting on Origami in Science, Mathematics and Education, Vol. II (2014), p. 537.
[36] Y. Miyamoto, in Origami 7: The 7th International Meeting on Origami in Science, Mathematics and Education, Vol. III (2018), p. 731.

[37] See Supplemental Material at http://ink.aps.org/supple mental/10.1103/PhysRevApplied.17.L021004 for details of experiments and FEA simulation, additional experimental and FEA data, theoretical argument on the rigid foldability of RES, and Movie S1, which includes Refs. [36,40-43].

[38] A. Ghassaei, E. Demaine, and N. Gershenfeld, Fast, interactive origami simulation using GPU computation, in Origami 7, Vol.4 (2018), p. 1151.

[39] To be exact, our threefold symmetric RES is tristable. It can pop out either upwards or downwards, where resulting structures are mirror-symmetric, with their chiralities being opposite, and all the vally and mountain folds being interchanged. As those reflection-symmetric strucutres are mechanically identical, we describe in this study our RES as being bistable.

[40] J. C. Maxwell, On the calculation of the equilibrium and stiffness of frames, Philos. Mag. 27, 294 (1864).

[41] C. R. Calladine, Buckminster Fuller tensegrity structures and Clerk Maxwell rules for the construction of stiff frames, Int. J. Solids Struct. 14, 161 (1978).

[42] T. C. Lubensky, C. Kane, X. Mao, A. Souslov, and K. Sun, Phonons and elasticity in critically coordinated lattices, Rep. Prog. Phys. 78, 073901 (2015).

[43] T. Tachi, in Proceedings of the International Association for Shell and Spatial Structures (IASS) Symposium, Vol. 12 (2010).

[44] Owning to small (but unavoidable) imperfections in the fabrication, the rotational axis of the hub and of the base did not match completely, and some strains remained in the both stable configurations, which is unimportant to our main results.

[45] B. Audoly and Y. Pomeau, Elasticity and Geometry: from Hair Curls to the Non-Linear Response of Shells (Oxford University Press, New York, 2010).

[46] For a planar buckling of a uniform rod, we have $\alpha=$ $\pi^{2}, 4 \pi^{2}$ and $\pi^{2} / 4$, for hinged, clamped, and free boundary conditions, respectively. For more general boundary conditions and specific strip geometry including our case, the exact value of $\alpha$ will also fall within the range of $\pi^{2} / 4-4 \pi^{2}$.

[47] B. Liu, J. L. Silverberg, A. A. Evans, C. D. Santangelo, R. J. Lang, and I. Cohen, Topological kinematics of origami metamaterials, Nat. Phys. 14, 811 (2018).

[48] Z. Chang, T. D. Ta, K. Narumi, H. Kim, F. Okuya, D. Li, K. Kato, J. Qi, Y. Miyamoto, K. Saito, and Y. Kawahara, in Proceedings of the $2020 \mathrm{CHI}$ Conference on Human Factors in Computing Systems (CHI'20) (2020), p. 1. 